needlessly hurt the feelings of the person criticised. I wrote with all seriousness. I believe that Professor Ladd's utterances are conflicting, and it seems to me that anyone who points out this fact does him a real service.

University of Pennsylvania.

George Stuart Fullerton.

\title{
VISCERAL DISEASE AND PAIN.
}

In a series of papers published between I 893 and I 896 , Dr. Henry Head has treated 'Disturbances of Sensation with Especial Reference to the Pain of Visceral Disease." The starting point of his investigation is the well-known fact that visceral disorders are frequently accompanied by cutaneous tenderness, the pain occasioned by organic disturbance being ' referred' by the patient to an area on the surface of the body. Dr. Head has carefully mapped out these areas, designating in his first paper those which lie below the first dorsal segment, and in his second paper those which are found on the head and neck. His third paper deals, not with the topography of the areas, but with the pain caused by diseases of various organs. His report contains a yast amount of clinical evidence interspersed with theoretical considerations. The value of his contributions to pathology, anatomy and physiology has been duly recognized. And, quite naturally, the results which he obtained and which certainly throw light on an intricate problem, have been pressed, with some eagerness, into the service of psychological theory. ${ }^{2}$ Whether there are separate nerves for pain is a question which cannot be fully discussed here; but the evidence in favor of the affirmative view supplied by Head's research is such as to deserve examination.

It seems to be fairly established that in cases of visceral disease certain skin-areas are affected in such a way that they show increased tenderness, increased reflexes, and one or more maximal points to which the pain is referred and to which the tenderness is limited as the disturbance subsides. The tenderness can be tested by applying a pin to the sensitive area, in which case the rounded head causes as sharp a sensation as the point causes on normal surfaces, while the application of the point gives rise to excessive pain. Quantitative data as to the amount or duration of pressure are not furnished in Dr. Head's report.

IBrain, XVI., I893, p. I; XVII., 1894, p. 339; XIX., I896, p. I53.

${ }^{8}$ Pain Nerves. Herbert Nichols, PsY. Rev., May, 1896, p. 309. 
What is, perhaps, of greater importance, he has shown that the eruptions in Herpes Zoster occupy areas which have the same distribution and the same maxima as the areas of tenderness in visceral disease.

From these statements it must not be inferred that the topography of the areas of tenderness is a simple or easy affair. At times only the maxima can be determined; in nearly all cases more than one area can be pointed out; and certain areas of the body which are rarely affected, appear, when they do become tender, in combination with others.

With these facts as a basis, Dr. Head proceeds by way of elimination to show the significance of the areas. They bear no relation to cortical distribution, nor do they correspond to the distribution of peripheral nerves. Do they represent the supply from posterior nerveroots? To this question a negative reply is given. The areas supplied from the roots overlap, whereas the areas of cutaneous tenderness and of herpetic eruption do not overlap. Hence the inference that each of these latter areas represents the supply from a single segment of the cord. And since the touch nerves issue from several segments and, in their distribution, overlap, it would seem to follow that there are separate paths for touch and for pain.

So much stress is laid, in deductions of this sort, upon the way in which the zones are mapped out, that one may be permitted to look more closely at the facts of distribution. To begin with, it must be noted that the proofs for the overlapping of the touch-areas and the proofs for the limitation of the pain-areas, are not of precisely the same character. Sherrington found that when, in the monkey, a single posterior root is divided, there is no absence of sensation; and Head, in some few cases, observed the same thing in man. The same area, therefore, must be supplied from several roots and fibres from these must interlace. But in mapping out the areas of tenderness, Head observed a large number of subjects, locating an area in this patient and another area in another patient and so on. His criterion is this: "If they overlapped to any considerable extent, like the areas of common sensation, the extent of skin covered when any one was present must necessarily be greater than that left unaffected when the areas on each side of it were tender. That is to say-supposing Nos. I and 3 were tender, the skin between their borders, which was unaffected, must of necessity be of smaller extent than that affected when No. 2 only was tender." Whatever be the accuracy of this method, it is obviously less direct than the method employed to demonstrate the overlapping of touch-zones. Dr. Head himself does not contend for an absolute definition of the areas of tenderness; he admits more 
than once that there is some overlapping, though this is slight as compared with the overlapping of zones supplied from the posterior roots. It is a ' difference of degree.' Another investigator, Dr. Mackenzie, is more emphatic. He tells us: "From the study of cases of Herpes Zoster and of the hyperæsthetic areas associated with visceral disease, I have come to the conclusion that there is distinct overlapping of the fields of cutaneous supply in individual nerve roots, of pathic, thermic and trophic fibres as well as of those of ordinary sensation." 1 This divergence suggests that further investigation may be necessary before the argument from overlapping as against sharp definition can be securely formulated.

Much depends, of course, upon what is meant by 'different areas of distribution.' Experiment has shown that on the skin there are points for heat and points for cold; whence it is reasonable to infer that there are separate paths for these two kinds of stimulation. If the areas in question were simply expansions of similar points, each having its special and exclusive function, the evidence in favor of separate nerves for touch and for pain would be strong. There might be irregularities in the distribution and different degrees of sensibility in the various areas; but once the work of mapping out had been accomplished, we would be able to indicate, for any given area, its particular function. It will hardly be claimed that our topography of the skin has attained this ideal accuracy, so far as zones for pain distinct from touch-zones are concerned. In particular, the results published by Dr. Head do not establish any such clear demarcation. Consequently, it is not in this strict sense that Dr. Nichols must be understood when he states as a fact that " the zones of distribution for pain, heat and trophic nerves cover markedly different fixed areas of the skin from the zones of distribution of the touch-nerves."

It is possible that we are exacting too much-insisting on proofs that will never be forthcoming. In fact, different areas of distribution may be conceived after a less rigorous fashion. Different functions might occupy in part the same area, though their respective zones have different boundaries. It might be shown, for instance, that in a total area which we will call $\mathrm{I} 2$, the zones $\mathrm{I}-9$ are sensible to tactile stimuli and the zones $3^{-12}$ are sensible to painful stimuli. In this case, we should say that the zones for touch overlap the zones for pain, or vice versa, without admitting that one touch-zone overlaps another touchzone, or that the zones for pain overlap one another. Under such conditions, the argument for separate pain nerves would be a more

I Brain, XVI., 1893 , p. 349. 
labored one. Nevertheless, it would have a weight of its own-provided that areas of this sort could be marked off on the normal subject.

There is more reason for doubt where difference of distribution hinges upon a change from normal to abnormal conditions. The argument might then take on several forms, one of which may be mentioned simply to show that the phrase 'markedly different fixed areas' needs careful interpretation. From the diagrams furnished by Dr. Head, it appears that the areas of cutaneous tenderness in visceral disease are quite large, extending in some cases in broad bands around the body or along the limbs. If the 'fixedness' could possibly imply that these areas are in all cases, normal no less than pathological, reserved for pain, the markedly different areas for touch would be rather limited. The likelihood of such a misconception is not great where one merely compares the normal condition of any organ with its abnormal condition. But at present we are dealing not with local, but with referred pain. The disease is visceral; the skin is supposed to be normal - or at least to be affected in only a roundabout way. Accordingly, one might infer, in consequence of the markedly different areas, that considerable portions of the skin are set apart for painful stimulation, and that they enter upon this function when the necessary condition, visceral disorder, is realized.

A more plausible form of the argument: areas supplied from the posterior roots and serving the function of touch overlap in normal conditions, whereas, in visceral disease, areas are marked off which serve the function of pain and do not overlap. This brings us in view of the question whether the same fibres which, under normal conditions transmit tactile stimulation, do not serve as pain-paths in visceral disease. It will be remembered that, according to Obersteiner's observations, there are diseases in which tactile stimulation of one portion of the skin gives rise to a sensation which the patient localizes in another portion. This allocheiria is due to a lesion in the central nervous system. According to Dr. Head, "the phenomena of allocheiria and of referred pain in visceral disease are in nature and explanation essentially the same. Both depend for their appearance upon the law that where a painful stimulus is applied to a part of lower sensibility in close central connection with a part of much greater sensibility, the pain produced is felt in the part of higher sensibility rather than in the part of lower sensibility to which the stimulus was actually applied." This explanation bears directly upon the transferred localization of tactile and painful stimuli; but it will also account, I think, for the painful feeling itself. As a result of visceral disease there is a height- 
ened excitability at the point of central connection; hence the exaggerated reflexes which characterize the affected skin-areas. A stimulus which, under normal conditions, would produce only a sensation of touch, passes into the modified center and is referred, in painful phase, to the stimulated area. Or again, stimuli originating in an internal organ and producing ordinarily unconscious reflexes, are referred, in the altered condition of the cord, either to a superficial area or to the diseased region itself, as is the case when the serous cavities of the body are affected. On this hypothesis, the difference between parts of higher sensibility and parts of lower sensibility might, to some extent, be explained. The higher sensibility of superficial areas is more easily understood if we suppose the same fibres to conduct tactile and painful stimulation. Frequency of tactile stimulation and transmission would increase the sensibility of the skin areas, whereas, on the hypothesis of separate paths, it is difficult to see why the skin should be more sensible and why the pain should be referred to it rather than to the seat of disease.

Should this view prove correct, the difference of distribution would cease to be a primary factor in the problem. The effect of stimulation would depend, not so much on the number of segments in the cord that it reaches, as on the condition of any or of all the segments. Whether the areas of cutaneous tenderness in visceral disease are sharply defined or distinctly overlap, is at best an open question. Its final settlement, no doubt, will be hastened by painstaking research along the lines of Dr. Head's investigation. One may fully appreciate his work without feeling bound to declare, in the words of Dr. Nichols, that it 'must set this dispute at rest forever.'

E. A. Pace.

Catholic University, Washington. 